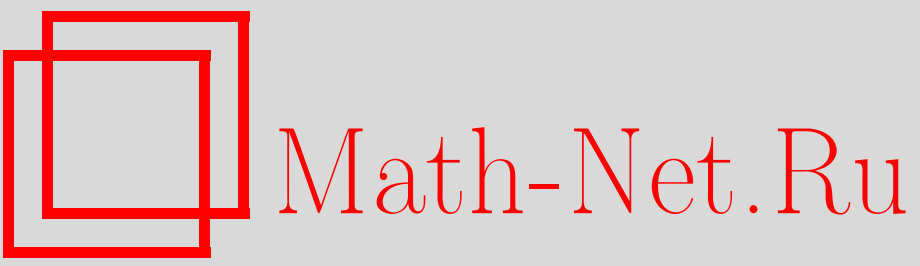

K. Конно, Х. Какухата, Новый тип решений с растяжением локального индукционного уравнения, возбужденных первоначально растянутыми вихревыми нитями, ТМФ, 2005, том 144, номер 2, 354-363

DOI: https://doi.org/10.4213/tmf1861

Использование Общероссийского математического портала Math-Net.Ru подразумевает, что вы прочитали и согласны с пользовательским соглашением

http: //www.mathnet.ru/rus/agreement

Параметры загрузки:

IP : 18.234 .197 .8

26 апреля 2023 г., $06: 23: 29$ 
ТЕОРЕТИЧЕСКАЯ

И МАТЕМАТИЧЕСКАЯ

ФИЗИКА

Том 144, № 2

август, 2005

(C) 2005 г.

К. Конно*, Х. Какухата ${ }^{\dagger}$

\section{НОВЫЙ ТИП РЕШЕНИЙ С РАСТЯЖЕНИЕМ ЛОКАЛЬНОГО ИНДУКЦИОННОГО УРАВНЕНИЯ, ВОЗБУЖДЕННЫХ ПЕРВОНАЧАЛЬНО РАСТЯНУТЫМИ ВИХРЕВЫМИ НИТЯМИ}

Решается задача Коши для локального индукционного уравнения; начальные данные представляют собой растянутую вихревую нить. Новый тип решений с растяжением, возникающих при численном моделировании, получен аналитически.

Ключевые слова: локальное индукционное уравнение, вихревые нити, растянутые нити.

\section{1. ВВЕДЕНИЕ}

Динамика эластичной струны (нити), демонстрирующей растяжение и/или стягивание, является очень интересной задачей. Нерастянутая струна не описывается линейным уравнением, а подчиняется нелинейному уравнению [1]. С другой стороны, растянутая струна также описывается нелинейным уравнением [2], которое обладает интересными уединенными решениями. В своих недавних работах авторы исследовали движение растяжимых струн в трехмерном пространстве с точки зрения того, как ведет себя растянутая струна. В работе [3] был проведен численный анализ движения вихревой нити в случае, когда нить подчиняется локальному индукционному уравнению (ЛИУ) с поправками, порождаюшими ее растяжение. В работе [4] было рассмотрено интегрируемое уравнение, учитываюшее два нелинейных эффекта, растяжение и стягивание. Это уравнение обладает солитонным решением, имеюшим форму растяжимой петли. Было показано, что первопричиной устойчивости солитона является конкуренция двух упомянутых эффектов.

В настоящей работе рассматривается (численно и аналитически) движение растянутых вихревых нитей, описываемых ЛИУ

$$
\mathbf{r}_{t}=\mathbf{r}_{s} \times \mathbf{r}_{s s}
$$

* Department of Physics, College of Science and Technology, Nihon University, Tokyo 101-8308, Japan. E-mail: konno@phys.cst.nihon-u.ac.jp

${ }^{\dagger}$ Toyama University, Toyama 930-8555, Japan 
где $t$ - время, $s$ - параметр вдоль нити и $\mathbf{r}$ - радиус-вектор,

$$
\mathbf{r}=(X, Y, Z)
$$

ЛИУ часто переписывают с использованием репера Френе в виде

$$
\mathbf{r}_{t}=\kappa \mathbf{b},
$$

где $\mathbf{b}$ - вектор бинормали, $\kappa$ - кривизна. Заметим, что при использовании этого репера касательньй вектор должен удовлетворять условию $|\mathbf{t}|=1$. Однако уравнение (1), как видно, дает $\left(\mathbf{r}_{s}^{2}\right)_{t}=0$, поэтому мы имеем условие

$$
\mathbf{r}_{s}^{2}=f(s)
$$

Следовательно, величина $f(s)$ не обязана быть константой, но может быть функцией переменной $s$. Если $f(s)$ зависит от $s$, то (3) не эквивалентно (1).

Нас интересует проблема временно́й эволюции вихря, движение которого задается ЛИу (1), в случае, когда в качестве начального условия выбирается локально растянутый вихрь, т.е. $\left|\mathbf{r}_{s}\right|$ есть функция от $s$. Одной из причин, побудивших нас провести численное моделирование, является желание исследовать различие между уравнениями (1) и (3). Мы рассматриваем три вида начальных условий: 1) растянутый вихревой солитон в трехмерном пространстве, 2) вихревая нить петлевого типа в двумерном пространстве и 3) вихревая нить кускового типа в двумерном пространстве.

Используя определенное в разделе 2 локальное растяжение, мы наблюдаем следующее. В случае начальных условий первого вида, после того как вихревой солитон удалится на большое расстояние, возникает растянутая нить нового типа, которая создает растяжение в той же области пространства, где оно было локализовано первоначально. В случае начальных условий второго вида вихревая нить петлевого типа врашается без распространения в пространстве, так что растяжение нити с течением времени остается локализованным в одной и той же области. В случае начальных условий третьего вида кусок нити быстро исчезает и вместо нее возникает растянутая нить нового типа, отвечающая первому случаю.

Для того чтобы объяснить результаты численного моделирования, мы ищем некоторый класс решений ЛИУ с помошью метода разделения переменных. Мы получаем зависяшее от времени решение, врашаюшееся в определенной области пространства, а также решение, не зависящее от времени. Последнее решение описывает динамику нити, у которой координаты $X$ и $Y$ в (2) принимают постоянные значения, а величина $Z-s$ имеет форму кинка, что и создает растяжение нити.

Работа организована следующим образом. Во втором разделе определяется локальное растяжение вихревой нити. В разделе 3 представлены результаты численного моделирования. В разделе 4 получены точные решения уравнения (1) с помошью метода разделения переменных. В последнем разделе обсуждаются основные результаты работы. 


\section{2. ОПРЕДЕЛЕНИЕ ЛОКАЛЬНОГО РАСТЯЖЕНИЯ}

Локальная длина $d l(s)$ вихревой нити задается формулой

$$
d l=\sqrt{(d X)^{2}+(d Y)^{2}+(d Z)^{2}} .
$$

Используя параметр вдоль нити $s$, можно переписать последнюю формулу в виде

$$
d l(s)=\sqrt{\left(X_{s}\right)^{2}+\left(Y_{s}\right)^{2}+\left(Z_{s}\right)^{2}} d s .
$$

Тогда локальное растяжение $l_{s}$ определяется формулой

$$
l_{s}=\frac{d l}{d s}=\sqrt{\left(X_{s}\right)^{2}+\left(Y_{s}\right)^{2}+\left(Z_{s}\right)^{2}} .
$$

Нить без растяжения отвечает случаю $l_{s}=1$, нить с растяжением - случаю $l_{s}>1$, а нить со стягиванием - случаю $l_{s}<1$. Условие (4) предполагает, что сушествуют растянутые или стянутые вихревые нити.

Как будет видно из дальнейших рассуждений, такая мера локального растяжения дает мощное средство поиска новых мод, в то время как поиск мод на основе наблюдения временно́й эволюции профилей нитей представляет собой очень трудную задачу.

\section{3. ЗАДАЧА КОШИ}

3.1. Растянутый вихревой солитон. Введем растянутый вихревой солитон, динамика которого описывается уравнениями

$$
\begin{aligned}
X & =A \frac{\lambda_{\mathrm{I}}}{\lambda_{R}^{2}+\lambda_{\mathrm{I}}^{2}} \sin (2 \Omega+\delta) \operatorname{sch}(2 \Theta+\epsilon), \\
Y & =-A \frac{\lambda_{\mathrm{I}}}{\lambda_{R}^{2}+\lambda_{\mathrm{I}}^{2}} \cos (2 \Omega+\delta) \operatorname{sch}(2 \Theta+\epsilon), \\
Z & =s-\frac{\lambda_{\mathrm{I}}}{\lambda_{R}^{2}+\lambda_{\mathrm{I}}^{2}} \operatorname{th}(2 \Theta+\epsilon),
\end{aligned}
$$

где $A$ - коэффишиент, характеризуюший растяжение. Если $A=1$, то солитон (8) есть просто точное решение уравнения (1) в отсутствие растяжения. Величины $\Omega, \Theta$ и постоянные $\delta, \epsilon$ задаются следуюшим образом:

$$
\begin{gathered}
\Omega=\lambda_{\mathrm{R}} s-\omega_{\mathrm{R}} t, \quad \Theta=\lambda_{\mathrm{I}} s-\omega_{\mathrm{I}} t, \\
\operatorname{tg} \delta=-\frac{2 \lambda_{\mathrm{R}} \lambda_{\mathrm{I}}}{\lambda_{\mathrm{R}}^{2}-\lambda_{\mathrm{I}}^{2}}, \quad \epsilon=-\frac{1}{2} \ln \frac{\left|c_{0}\right|^{2}}{4 \lambda_{\mathrm{I}}^{2}},
\end{gathered}
$$

где $c_{0}$ - константа. Волновое число $\lambda=\lambda_{\mathrm{R}}+i \lambda_{\mathrm{I}}$ и частота $\omega=\omega_{\mathrm{R}}+i \omega_{\mathrm{I}}$ связаны дисперсионным соотношением

$$
\omega=2 \lambda^{2} .
$$

Локальное растяжение дается выражением

$$
l_{s}^{2}=1+\frac{4 \lambda_{\mathrm{I}}^{2}}{\lambda_{\mathrm{R}}^{2}+\lambda_{\mathrm{I}}^{2}}\left(A^{2}-1\right)\left[\operatorname{sch}^{2}(2 \Theta+\epsilon)-\frac{\lambda_{\mathrm{I}}^{2}}{\lambda_{\mathrm{R}}^{2}+\lambda_{\mathrm{I}}^{2}} \operatorname{sch}^{4}(2 \Theta+\epsilon)\right] .
$$



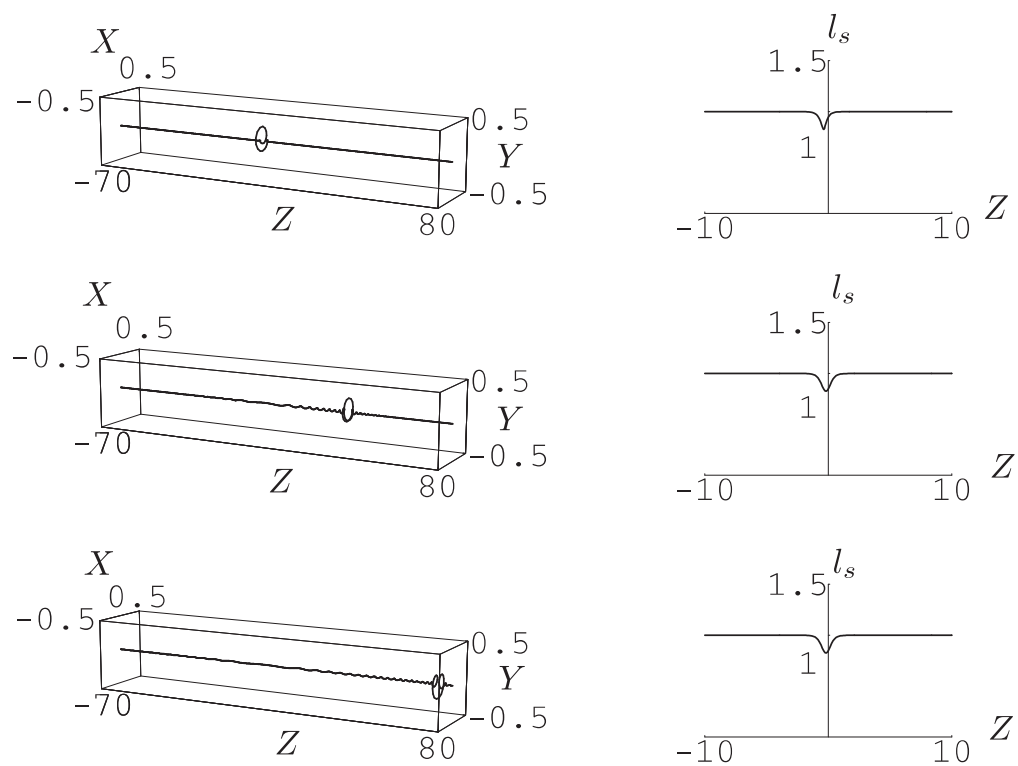

Рис. 1. Временна́я эволюция (слева) и соответствующее локальное растяжение (справа) растянутого вихревого солитона в моменты времени $t=0,6,12$ при $A=0.8$ и $\lambda=1.5+i$.

Если $A=1$, то растяжение отсутствует, при $A>1$ в (8) имеет место локальное растяжение, а при $A<1$ - стягивание.

Вычисления производились для трех значений $A=1,0.8,1.2$ при $\lambda=1.5+i$ и $c_{0}=1$. На рис. 1 представлены профили и соответствующие локальные растяжения вихрей в моменты времени $t=0,6,12$ для случая $A=0.8$. Мы видим, что если вихревая нить изначально растянута, то вихревой солитон движется вдоль луча, однако область растяжения остается на одном и том же месте, как "замороженная". Чтобы провести подробньй анализ растяжения нити, на рис. 2 представлено поведение каждой из ее координат $X, Y, Z$ около точки $s=0$ в случае $A=0.8$ при больших временах. Видно, что после того как вихревой солитон покинул рассматриваемую область, можно наблюдать возбуждение моды с постоянными значениями координат $X, Y$ и величиной $Z-s$, имеюшей форму кинка в области первоначальной локализации растяжения. Этот не зависящий от времени кинк создает растяжение нити. Сходная ситуация имеет место и в случае $A=1.2$.

3.2. Вихревая нить петлевого типа. В качестве начальных условий рассмотрим двумерную вихревую нить петлевого типа

$$
\begin{aligned}
& X=0, \\
& Y=A \operatorname{sch} k s, \\
& Z=s-\operatorname{th} k s .
\end{aligned}
$$

Коэффипиент $A$ задает параметр растяжения. Локальное растяжение дается выражением

$$
l_{s}^{2}=1+\left(A^{2} k^{2}-2 k\right) \operatorname{sch}^{2} k s-k^{2}\left(A^{2}-1\right) \operatorname{sch}^{4} k s .
$$



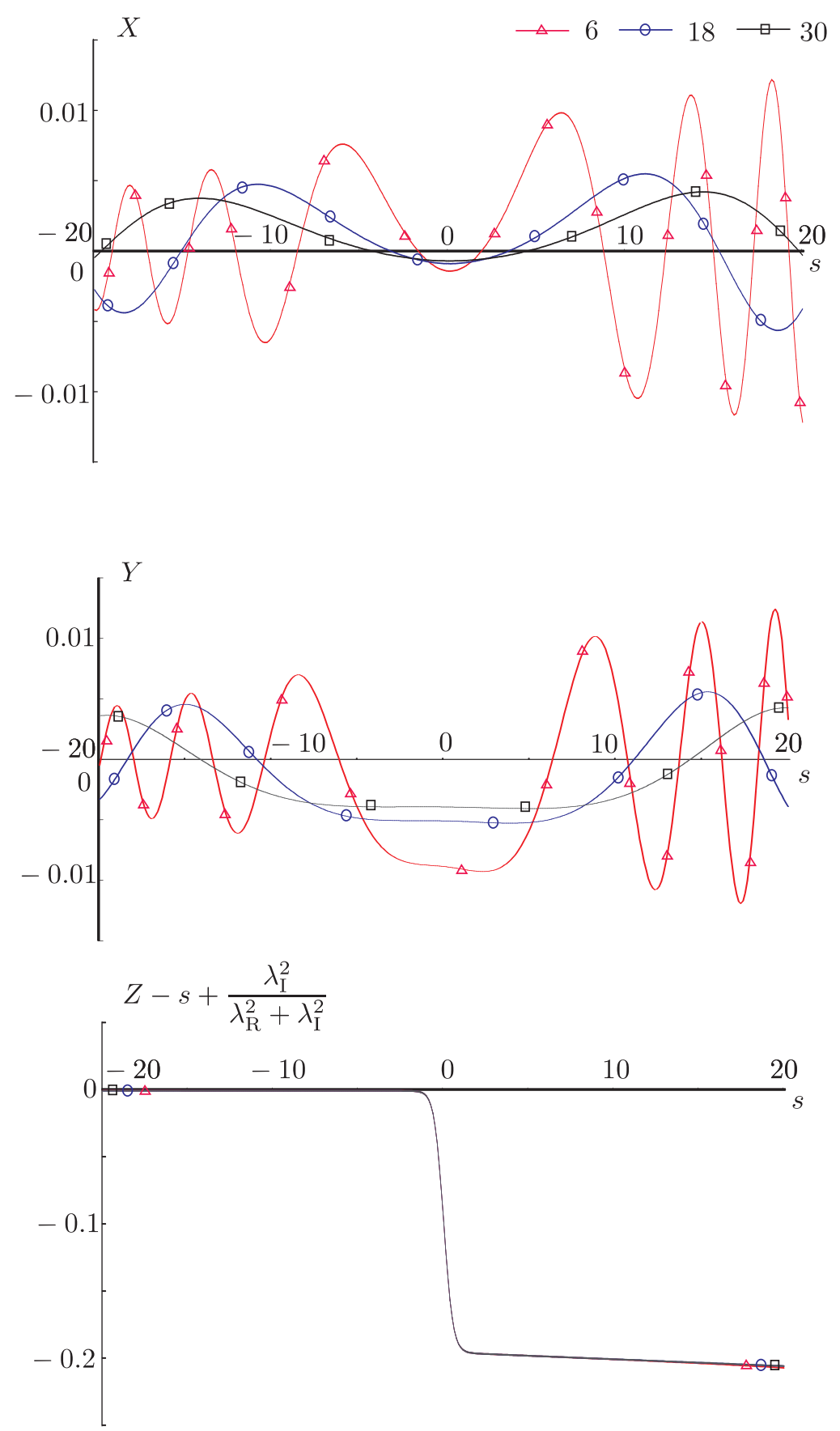

Рис. 2. Временна́я эволюция растянутого вихревого солитона в области $-20<s<20$ в моменты времени $t=6,18,30$ при $A=0.8$. 

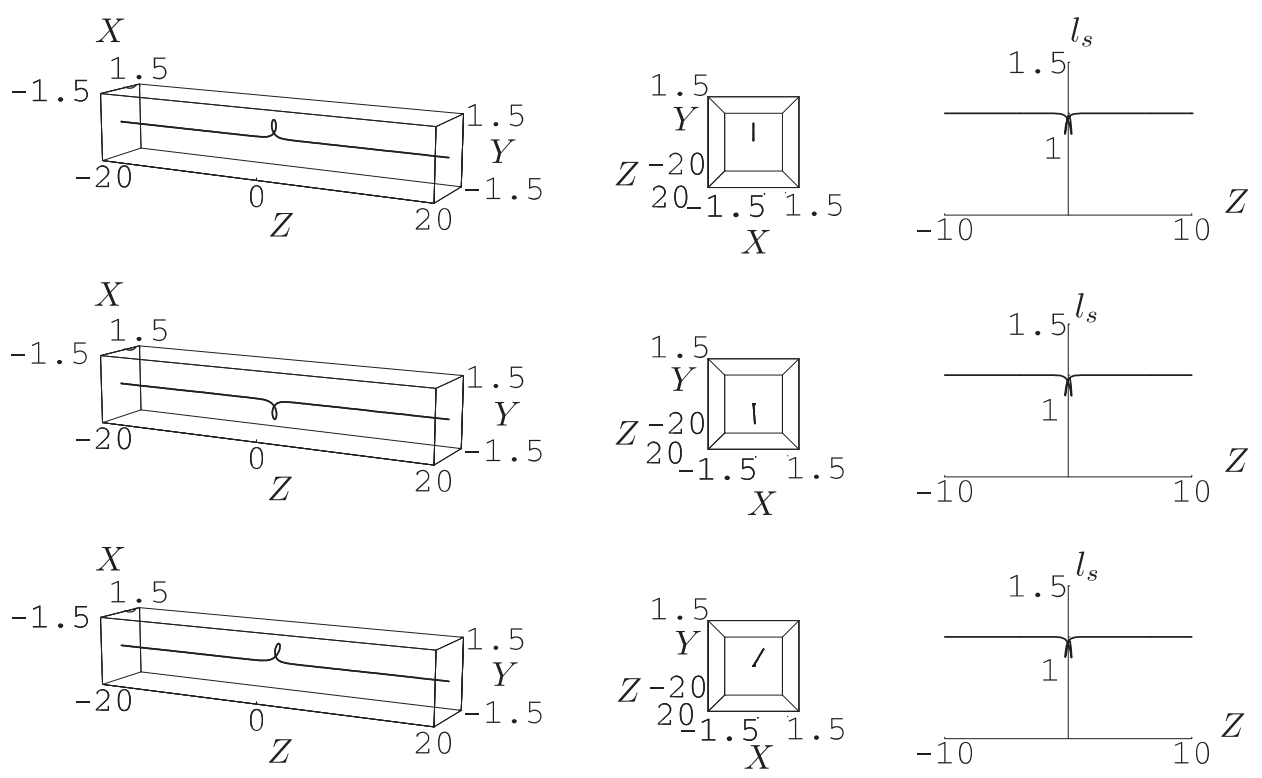

Рис.3. Временна́я эволюция (слева), вид сверху (в центре) и локальное растяжение (справа) вихря петлевого типа в моменты времени $t=0,6,12$ при $A=0.8$ и $k=2$.

Вычисления производились при $A=0.8,1.2$ и $k=2$; на рис. 3 представлены результаты для случая $A=0.8$. В обоих случаях петля врашается в определенной области пространства с постоянной угловой скоростью как "пришпиленная", так что область растяжения неподвижна ("заморожена") в пространстве. Вид сверху показывает, что форма петли в случае $A=0.8$ не испытывает деформаций.

3.3. Вихревая нить кускового типа. Двумерная вихревая нить кускового типа задается следуюшим образом:

$$
\begin{aligned}
X & =0, \\
Y & =A \operatorname{sch} k s, \\
Z & =s .
\end{aligned}
$$

Локальное растяжение дается выражением

$$
l_{s}^{2}=1+k^{2} A^{2} \operatorname{sch}^{2} k s \operatorname{th}^{2} k s
$$

На рис. 4 представлены результаты численного моделирования для случая $A=1$, $k=2$. С течением времени кусковая часть нити становится малой и наблюдается испускание малых вращающихся волн, которые распространяются в обоих направлениях. Отметим, что направления вращения испускаемых волн, распространяющихся в положительном и отрицательном направлении оси $Z$, противоположны, так что момент импульса сохраняется [5], при этом первоначально он равен нулю. На рис. 5 представлено поведение солитона около точки $s=0$ при больших временах. Для величины $Z-s$ наблюдается небольшое плато в окрестности точки $s=0$, которое дает два пика локального растяжения на рис. 4 , "замороженные" в окрестности $s=0$. Наблюдается также 

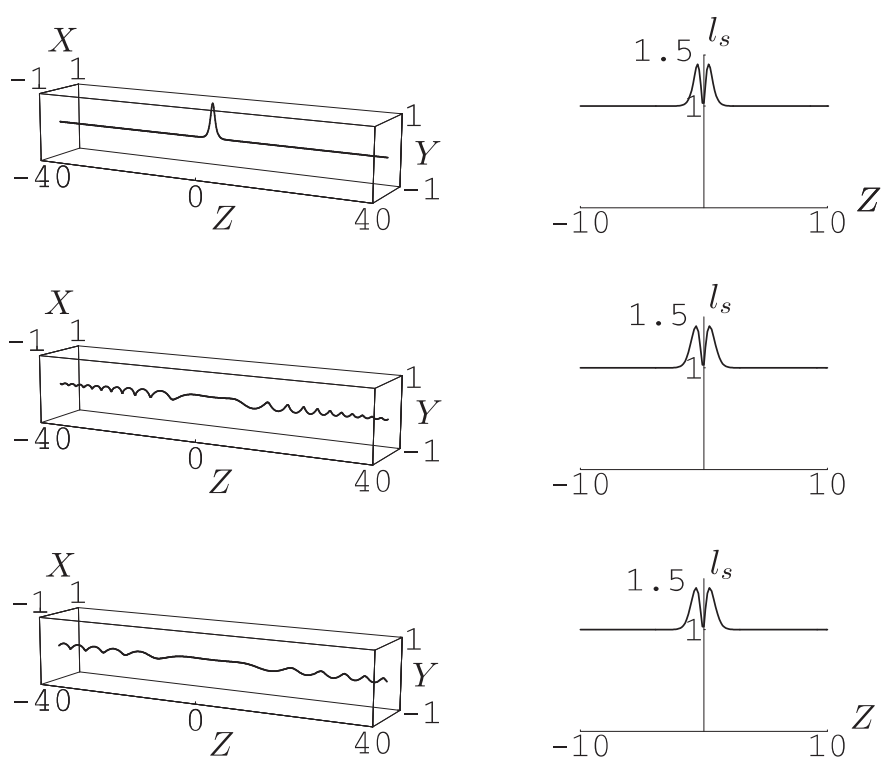

Рис. 4. Временна́я эволюция (слева) и соответствующее локальное растяжение (справа) вихря кускового типа в моменты времени $t=0,6,12$ при $A=1$ и $k=2$.

возбуждение моды с постоянными значениями координат $X$ и $Y$. Эта мода с двумя кинками очень похожа на приведенную в п. 3.1 моду с одним кинком.

\section{4. ТОЧНЫЕ РАСТЯНУТЫЕ РЕШЕНИЯ}

Численные расчеты показывают, что сушествуют точные решения ЛИУ (1) нового типа. Используем метод разделения переменных: будем искать решения в виде

$$
\begin{aligned}
X(s, t) & =T_{X}(t) W_{X}(s), \\
Y(s, t) & =T_{Y}(t) W_{Y}(s), \\
Z(s, t) & =Z(s) .
\end{aligned}
$$

При этом, основываясь на результатах проведенного численного моделирования, мы предполагаем, что функция $Z$ зависит только от переменной $s$. Подставляя (16) в (1), получаем следуюшие уравнения: для временно́й части решения

$$
\begin{aligned}
T_{X, t} & =c_{1} T_{Y}, \\
T_{Y, t} & =c_{2} T_{X} ;
\end{aligned}
$$

для пространственной части решения

$$
\begin{aligned}
& W_{Y, s} Z_{s s}-W_{Y, s s} Z_{s}=c_{1} W_{X}, \\
& Z_{s} W_{X, s s}-Z_{s s} W_{X, s}=c_{2} W_{Y}, \\
& W_{X, s} W_{Y, s s}-W_{X, s s} W_{Y, s}=0,
\end{aligned}
$$

где $c_{1}$ и $c_{2}-$ константы. 

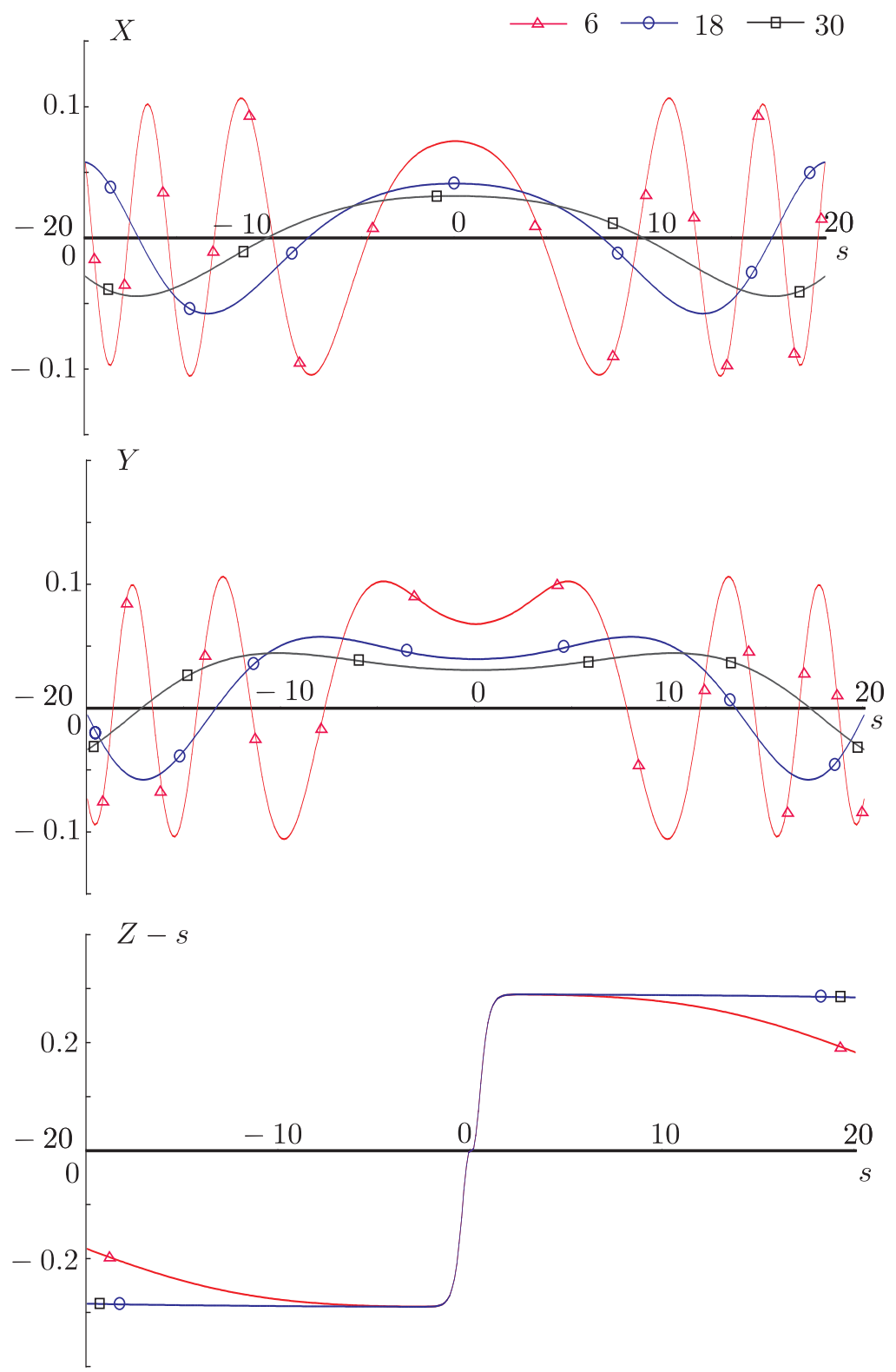

Рис. 5. Временна́я эволюция вихря кускового типа в области $-20<s<20$ в моменты времени $t=6,18,30$ при $A=0.8$.

4.1. Врашающееся петлевое решение. Для того чтобы объяснить поведение врашаюшейся вихревой нити петлевого типа из раздела 3.2 , будем искать решение в следуюшем виде:

$$
\begin{aligned}
& W_{X}(s)=A_{1} \operatorname{sch} k s, \\
& W_{Y}(s)=A_{2} \operatorname{sch} k s,
\end{aligned}
$$




$$
Z(s)=s-\text { th } k s
$$

Подставляя эти равенства в уравнения (18), получаем

$$
k=2, \quad c_{1} c_{2}=-16, \quad A_{2}=-\frac{c_{1}}{4} A_{1} .
$$

Определим временну́ю зависимость начальными условиями (12), так что при $t=0$ решение совпадает с вихревой нитью петлевого типа в начальном положении. Тогда

$$
\begin{gathered}
X=-A \sin 4 t \operatorname{sch} 2 s, \\
Y=A \cos 4 t \operatorname{sch} 2 s, \\
Z(s)=s-\operatorname{th} 2 s
\end{gathered}
$$

где $A$ - константа. Поведение построенного точного решения идентично поведению вихревой нити петлевого типа: мы наблюдаем врашение в определенной области пространства, и область растяжения остается на одном месте.

4.2. Не зависящее от времени решение. Для того чтобы понять особенности мод, найденных в пп. 3.1 и 3.3, будем искать не зависяшие от времени решения при $c_{1}=0$, $c_{2}=0$ в уравнениях (17). Положим

$$
\begin{gathered}
W_{X}(s)=A_{1} \\
W_{Y}(s)=A_{2}, \\
Z(s)=s+Z_{0}+\sum_{i=1}^{n} B_{i} \operatorname{th} k\left(s+s_{0 i}\right),
\end{gathered}
$$

где $A_{1}, A_{2}, Z_{0}, B_{i}$ и $s_{0 i}$ - константы, $n$ - число кинков для величины $Z-s$, найденное в ходе численного моделирования.

Рассмотрим сначала вихревое солитонное решение (8). Как показано на рис. 2, в случае $A=0.8$ по мере увеличения времени значения координат $X$ и $Y$ стремятся к постоянным величинам, а величина $Z-s$ обнаруживает один кинк вблизи точки $s=0$, который создает локальное растяжение $l_{s}$. Константы $A_{1}$ и $A_{2}$ соответствуют величинам $X$ и $Y$, которые экстраполируются функцией $a_{0}+a_{1} / t+a_{2} / t^{2}$. Получаем

$$
\begin{gathered}
X=-0.00053 \\
Y=-0.0031, \\
Z=s+0.31-0.10(1+\text { th } 1.7 s) .
\end{gathered}
$$

Аналогичным образом можно определить $X, Y$ и $Z$ в случае $A=1.2$ :

$$
\begin{gathered}
X=-0.00086 \\
Y=0.0025 \\
Z=s+0.31+0.093(1+\operatorname{th} 1.7 s) .
\end{gathered}
$$


Эти не зависящие от времени решения объясняют возникновение мод, возбуждаемых вихревыми солитонными решениями с растяжением.

Рис. 5 показывает, что для вихревой нити кускового типа из раздела 3.3 значения координат $X$ и $Y$ стремятся к постоянным величинам, а величина $Z-s$ имеет два кинка. В таком случае выражение для $Z$ в $(22)$ должно содержать два члена $(n=2)$. Предполагая, что асимптотическое поведение величин $X$ и $Y$ имеет прежний вид $a_{0}+a_{1} / t+a_{2} / t^{2}$, получаем

$$
\begin{gathered}
X=0.016 \\
Y=0.016 \\
Z=s+0.15(\operatorname{th} 2.8(s-0.5)+\operatorname{th} 2.8(s+0.5)) .
\end{gathered}
$$

Это решение хорошо описывает характерные особенности моды, возбуждаемой вихревой нитью кускового типа.

\section{5. ЗАКЛЮЧЕНИЕ}

Итак, исходя из локальной длины нити, мы определили локальное растяжение. Далее, были рассмотрены результаты численного моделирования динамики солитона для трех начальных случаев, а именно, растянутого вихревого солитона в трехмерном пространстве, вихревой нити петлевого типа в двумерном пространстве и вихревой нити кускового типа в двумерном пространстве.

Мы обнаружили, что возбуждаются моды новых типов. Одна мода соответствует постоянным значениям координат $X$ и $Y$ и обладающей кинками величине $Z-s$, а другая отвечает вращаюшемуся в определенном месте пространства петлевому решению.

Для того чтобы объяснить такое поведение этих мод, с помощью метода разделения переменных были построены точные решения с растяжением. Были найдены не зависящие от времени моды (23)-(25). Эти решения описывают “замораживание” локального растяжения для случаев вихревого солитона и вихревой нити кускового типа. Найдено также точное решение (22), которое описывает фиксацию центра врашения и "замораживание" локального растяжения для случая вихревой нити петлевого типа. Полученные аналитические решения способствуют пониманию результатов численного эксперимента.

Указанные решения не могут иметь касательного вектора единичной длины, поскольку величина $|\partial \mathbf{r} / \partial s|$ не является постоянной, а зависит от $s$. В таком случае ЛИУ (1) не эквивалентно уравнению (3). Уравнение (1) должно обладать более широким классом решений, чем уравнение (3).

\section{Список литературы}

[1] L. J. F. Broer. J. Eng. Math. 1970. V. 4. P. 195.

[2] V. Krylov, P. Rosenau. Phys. Lett. A. 1996. V. 217. P. 31.

[3] K. Konno, H. Kakuhata. Stretching of vortex filament with corrections. In: Nonlinear Physics: Theory and Experiment. II (Gallipoli, Lecce, Italy, 24 June - 3 July 2004). Eds. M. Ablowitz, M. Boiti, F. Pempinelli, B. Prinari. River Edge, NJ: World Scientific, 2003. P. 273.

[4] К. Конно, Х. Какухата. ТМФ. 2003. Т. 137. № 2. С. 201.

[5] Х. Kакухата, К. Конно. ТМФ. 2002. Т. 133. № 3. С. 419. 